\title{
Syringic Acid: Structural Elucidation and Co-crystallization
}

\author{
Rajesh Thipparaboina ${ }^{1}$, Sudhir Mittapalli ${ }^{2}$, Sowjanya Thatikonda ${ }^{3}$, Ashwini Nangia ${ }^{2,4}$, VGM \\ Naidu ${ }^{3}$, Nalini R Shastri ${ }^{1, *}$ \\ ${ }^{1}$ Solid State Pharmaceutical Research Group (SSPRG), National Institute of Pharmaceutical \\ Education and Research, Hyderabad, India \\ ${ }^{2}$ School of Chemistry, University of Hyderabad, Central University PO, Prof. C. R. Rao Road, \\ Hyderabad, India \\ ${ }^{3}$ Department of Pharmacology, National Institute of Pharmaceutical Education and Research, \\ Hyderabad, India \\ ${ }^{4}$ CSIR-National Chemical Laboratory, Pune, India.
}

\section{Supporting Information}

\section{Basic Pharmaceutical Terms used in the manuscript}

Angle of repose: Angle of repose is a characteristic related to interparticulate friction or resistance to movement between particles. Angle of repose is not an intrinsic property of the powder; i.e., it is dependent upon the method used to form the cone of powder. The following important considerations are raised in the existing literature ${ }^{1,2}$.

The peak of the cone of powder can be distorted by the impact of powder from above. By carefully building the powder cone, the distortion caused by impact can be minimized. The nature of the base upon which the powder cone is formed influences the angle of repose. It is recommended that the powder cone be formed on a "common base" which can be achieved by forming the cone of powder on a layer of powder. This can be done by using a base of fixed diameter with a protruding outer edge to retain a layer of powder upon which the cone is formed.

Capping: Capping occurs when the upper segment of the tablet separates from the main portion of the tablet \& comes off as a cap. Capping occurs either because air is entrapped in the tablet or too much elastic energy is stored after main compression ${ }^{3}$. Capping is one of the most common tablet defects and it can be simple to correct and proper diagnosis of the root cause of the capping problem is critical to correcting and eliminating capping. A key step to resolving capping is to increase dwell time. This will allow the particles more time to lock together. 


\section{Tables}

Table S1 HPLC method parameters

\begin{tabular}{|c|c|c|c|}
\hline Parameter & \multicolumn{3}{|l|}{ Details } \\
\hline Column & \multicolumn{3}{|c|}{$\mathrm{XBridge}^{\mathrm{TM}} 5 \mu, \mathrm{C}_{18}, 4.6 \times 100 \mathrm{~mm}$} \\
\hline Mobile phase & \multicolumn{3}{|c|}{$0.1 \%$ Formic acid buffer (FA) and Acetonitrile (ACN) } \\
\hline \multirow[t]{6}{*}{ Gradient program } & Time (min) & FA $(\%)$ & $\mathrm{ACN}(\%)$ \\
\hline & 1.00 & 95 & 5 \\
\hline & 2.00 & 80 & 20 \\
\hline & 3.00 & 10 & 90 \\
\hline & 5.00 & 95 & 5 \\
\hline & 6.00 & 95 & 5 \\
\hline Column temperature & \multicolumn{3}{|l|}{$25^{0} \mathrm{C}$} \\
\hline Sample temperature & \multicolumn{3}{|l|}{$25{ }^{0} \mathrm{C}$} \\
\hline$\lambda \max$ & \multicolumn{3}{|l|}{$275 \mathrm{~nm}$} \\
\hline Retention time $\left(\mathrm{R}_{\mathrm{t}}\right)$ & \multicolumn{3}{|l|}{$4.33 \mathrm{~min}$} \\
\hline Equation & \multicolumn{3}{|c|}{$Y=33057 x+52717$} \\
\hline Regression coefficient $\left(\mathrm{R}^{2}\right)$ & \multicolumn{3}{|l|}{0.9996} \\
\hline Calibration range & \multicolumn{3}{|l|}{$5-60 \mu \mathrm{g} / \mathrm{ml}$} \\
\hline
\end{tabular}

Table S2 Wells protocol for studying compressibility

\begin{tabular}{|l|c|c|c|}
\hline Sample amount & \multicolumn{3}{|c|}{$500 \mathrm{mg}+1 \%$ Magnesium stearate } \\
\hline Sample code & $\mathrm{A}$ & $\mathrm{B}$ & $\mathrm{C}$ \\
\hline Blending time & $5 \mathrm{~min}$ & $5 \mathrm{~min}$ & $30 \mathrm{~min}$ \\
\hline \multicolumn{4}{|c|}{ Compression using $13 \mathrm{~mm}$ die at $200 \mathrm{~kg} / \mathrm{cm}^{2}$ pressure } \\
\hline Dwell time & $2 \mathrm{~s}$ & $30 \mathrm{~s}$ & $2 \mathrm{~s}$ \\
\hline \multicolumn{4}{|c|}{ Equilibration in closed glass vials for 24 hour } \\
\hline \multicolumn{3}{|c|}{ Tablet crushing strength measurement } \\
\hline
\end{tabular}


Table S3 Observations from preliminary screening

\begin{tabular}{|c|c|c|c|}
\hline Coformer & LAG & SC & Observations \\
\hline Nicotinamide & + & $\begin{array}{l}\text { Single crystals } \\
\text { obtained }\end{array}$ & Successfully characterized and evaluated \\
\hline Urea & + & $\begin{array}{l}\text { Single crystals } \\
\text { obtained }\end{array}$ & Successfully characterized and evaluated \\
\hline Isoniazid & + & - & $\begin{array}{l}\text { New melting peaks observed but single crystals } \\
\text { couldn't be obtained. System under exploration to } \\
\text { study Hepatoprotective effects invivo. }\end{array}$ \\
\hline Piracetam & + & - & $\begin{array}{l}\text { Polymorphic transitions were observed during } \\
\text { thermal analysis. Fine needles were obtained and } \\
\text { were not suitable for structural analysis. System } \\
\text { under exploration to proceed further to study } \\
\text { neuroprotective effects in Alzheimer's model }\end{array}$ \\
\hline Levetiracetam & + & - & $\begin{array}{l}\text { Multiple endotherms and minor transitions observed. } \\
\text { Fine needles were obtained and were not suitable for } \\
\text { structural analysis. Melting patterns similar to } \\
\text { physical mixtures. }\end{array}$ \\
\hline Paracetamol & + & - & $\begin{array}{l}\text { Multiple endotherms were observed in thermal } \\
\text { analysis. Melting patterns similar to physical } \\
\text { mixtures. }\end{array}$ \\
\hline Pyrazinamide & - & - & $\begin{array}{l}\text { Melting patterns similar to physical mixtures. Both } \\
\text { components crystallized individually }\end{array}$ \\
\hline $5-\mathrm{FU}$ & + & - & Melting patterns similar to physical mixtures. \\
\hline
\end{tabular}

Table S4 Onset, peak, endset and enthalpy values

\begin{tabular}{|c|c|c|c|c|}
\hline Code & Onset $\left.\mathbf{(}^{\mathbf{0}} \mathbf{C}\right)$ & Peak $\left.\mathbf{(}^{\mathbf{0}} \mathbf{C}\right)$ & Endset $\left.\mathbf{(}^{\mathbf{0}} \mathbf{C}\right)$ & Enthalpy J/g) \\
\hline SYRA & 207.36 & 208.4 & 211.74 & 190.49 \\
\hline NCT & 127.63 & 128.66 & 133.03 & 191.73 \\
\hline U & 133.94 & 135.31 & 138.56 & 239.97 \\
\hline SNCT-E & 174.74 & 175.99 & 180.74 & 180.6 \\
\hline SU-EA-M & 167.55 & 168.01 & 172.12 & 201.52 \\
\hline
\end{tabular}


Table S5 Results from dissolution profile analysis

\begin{tabular}{|c|c|c|c|}
\hline Parameter & SYRA & SNCT-E & SU-EA-M \\
\hline $\mathbf{Q}_{\mathbf{5}} \mathbf{( \% )}$ & 67.99 & 22.67 & 28.16 \\
\hline $\mathbf{Q}_{\mathbf{1 0}} \mathbf{( \% )}$ & 71.07 & 30.72 & 29.39 \\
\hline $\mathbf{Q}_{\mathbf{1 5}} \mathbf{( \% )}$ & 73.98 & 41.14 & 50.51 \\
\hline $\mathbf{D E}_{\mathbf{5}} \mathbf{( \% )}$ & 33.90 & 11.33 & 14.00 \\
\hline $\mathbf{D E}_{\mathbf{1 0}} \mathbf{( \% )}$ & 51.70 & 19.00 & 21.40 \\
\hline $\mathbf{D E}_{\mathbf{1 5}} \mathbf{( \% )}$ & 58.60 & 24.60 & 27.50 \\
\hline DE (2h) (\%) & 85.50 & 75.30 & 78.70 \\
\hline AUC (2h) & 171.00 & 150.79 & 157.46 \\
\hline F2 & & 31.01 & 33.91 \\
\hline
\end{tabular}

Table S6 Inference from angle of repose studies $\left(n=6, \operatorname{Tan}^{-1}(h / r) \pm S D\right)$

\begin{tabular}{|c|c|c|c|c|}
\hline Code & Observed & \multirow{2}{*}{ Result } & \multicolumn{2}{|c|}{ inference } \\
\cline { 4 - 5 } & angle of repose & & Angle of repose & Flow \\
\hline SYRA & $47.72 \pm 0.4$ & Poor & $25-30$ & Excellent \\
\hline NCT & $30.96 \pm 0.31$ & Good & $31-35$ & Good \\
\hline U & $30.96 \pm 0.32$ & Good & $36-40$ & Fair \\
\hline SNCT-E & $38.65 \pm 0.42$ & Fair & $41-45$ & Passable \\
\hline SU-EA-M & $38.65 \pm 0.41$ & Fair & $46-55$ & Poor \\
\hline- & - & - & $56-65$ & Very poor \\
\hline- & - & - & $>66$ & Very very poor \\
\hline
\end{tabular}

Table S7 Inference Well's compressibility studies ( $n=3$, Hardness \pm SD) $C P=$ Capping

\begin{tabular}{|c|c|c|c|c|c|}
\hline Codes & A & B & C & Observation & Inference \\
\hline SYRA & $2.27 \pm 0.36$ & $1.8 \pm 0.5$ & $2.03 \pm 0.26$ & $\mathrm{~A}>\mathrm{C}>\mathrm{B}$ & Brittle \\
\hline $\mathrm{U}$ & $3 \pm 0.92$ & $5.5 \pm 0.65$ & $3.54 \pm 0.50$ & $\mathrm{~B}>\mathrm{A}>\mathrm{C}$ & Plastic \\
\hline NCT & $\mathrm{CP}$ & $3.5 \pm 0.6$ & $2.34 \pm 0.55$ & Capping & Elastic \\
\hline SNCT-E & $\mathrm{CP}$ & $1.36 \pm 0.47$ & $\mathrm{CP}$ & Capping & Elastic \\
\hline SU-EA-M & $\mathrm{CP}$ & $1.46 \pm 0.55$ & $2.17 \pm 0.26$ & Capping & Elastic \\
\hline
\end{tabular}


<smiles>NNC(=O)c1ccncc1</smiles>

Isoniazid<smiles>O=c1[nH]cc(F)c(=O)[nH]1</smiles>

5-Fluoro-Uracil

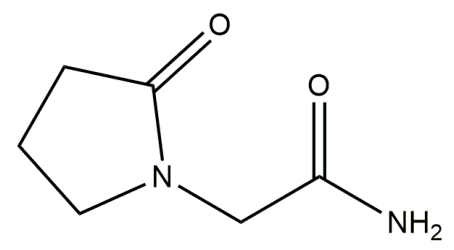

Piracetam<smiles>NC(=O)c1cccnc1</smiles>

Nicotinamide<smiles>COc1cc(C(=O)O)cc(OC)c1O</smiles>

Syringic acid

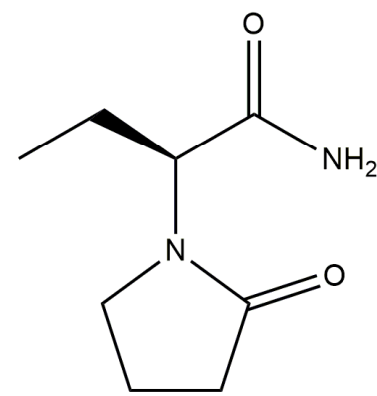

Levetiracetam

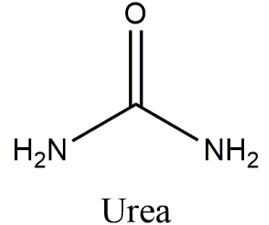<smiles>NC(=O)c1cnccn1</smiles><smiles>CC(=O)Nc1ccc(O)cc1</smiles>

Paracetamol

Figure S1 Chemical structures of the compounds studied 


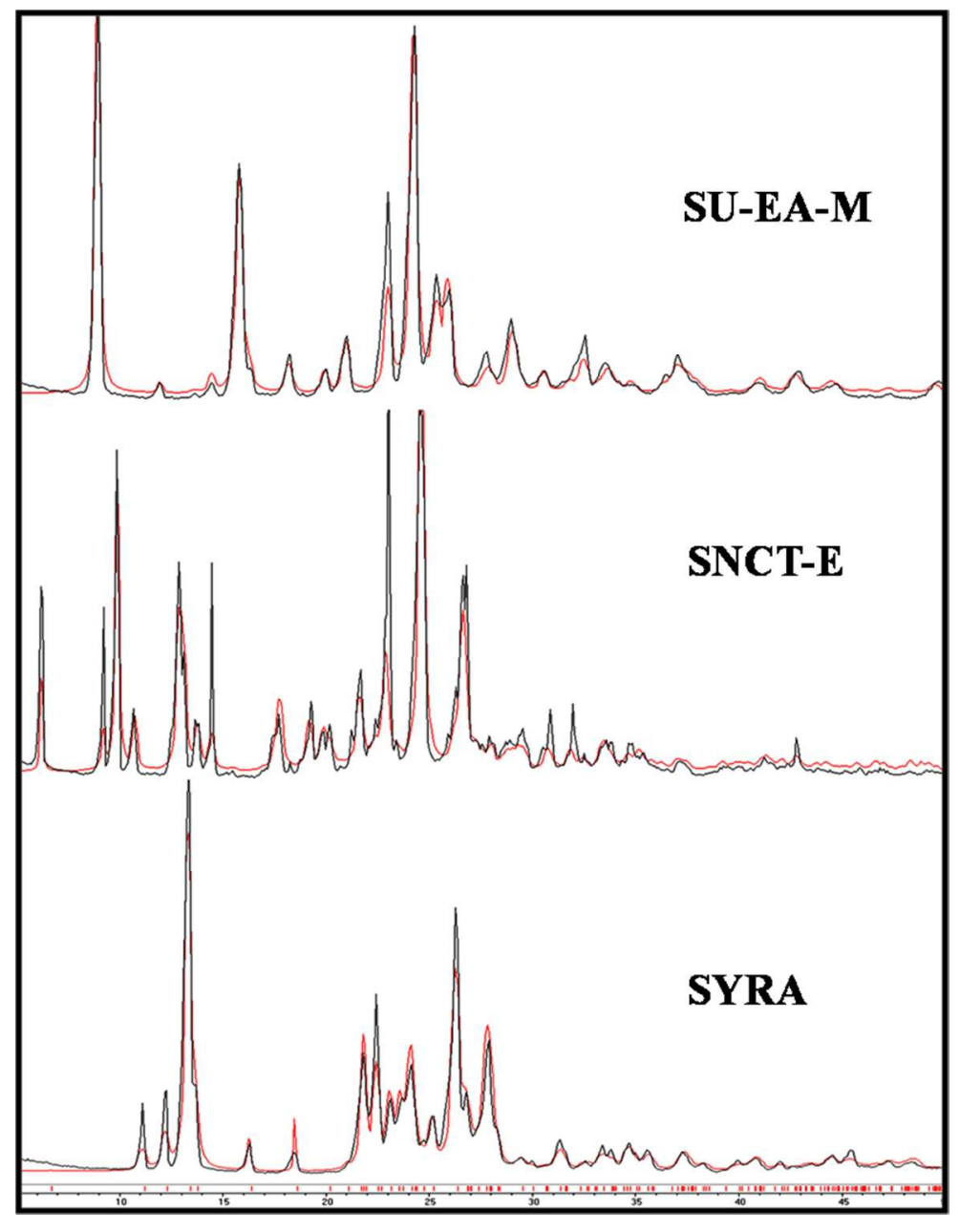

Figure S2 Overlay of calculated and experimental powder patterns.

\section{References}

1. R. L. Carr, Chem. Eng. , 1965, 72, 163-168.

2. R. B. Shah, M. A. Tawakkul and M. A. Khan, Aaps Pharmscitech, 2008, 9, 250-258.

3. C.-Y. Wu, B. Hancock, A. Mills, A. Bentham, S. Best and J. Elliott, Powder Technology, 2008, 181, 121-129. 